\title{
THE ANTERIOR CHAMBER ANGLE IS DIFFERENT IN DIFFERENT RACIAL GROUPS: A GONIOSCOPIC STUDY
}

\author{
YOUNGHYUN G. OH ${ }^{1}$, SILVANA MINELLI ${ }^{2}$, GEORGE L. SPAETH $^{3}$ and WILLIAM C. STEINMAN ${ }^{4}$ \\ Philadelphia, Pennsylvania
}

\begin{abstract}
SUMMARY
The purpose of the study was to determine racial and sexual differences and age-related changes in the anterior chamber angle to evaluate the reliability of a standardised gonioscopic grading system. The anterior chamber angle was studied prospectively in 291 patients who included Afro-Americans, Caucasians and Far East Asians. No statistically significant difference was found between the angle width of these three groups, but the iris joins the scleral wall more anteriorly in Asians, slightly more posteriorly in Afro-Americans, and most posteriorly in Caucasians $(p=0.03)$. The anterior chamber tends to be narrower in females $(p=0.002)$ and to become narrower with increasing age $(p<0.0001)$ in all ethnic groups. Refractive error significantly affects both the level of iris insertion $(p<0.003)$ and the angle width $(p<0.0001)$. The consistency of the angle grading system in this project is demonstrated by a close agreement between the examiners. It is concluded that the difference in the angle configuration may account for racial differences in the incidence of angle-closure glaucoma, this being least common in Caucasians, more common in Afro-Americans and most common in Asians.
\end{abstract}

Angle-closure glaucoma is reported to be more common among Eskimos than among Caucasians. ${ }^{1-7}$ However, there have been no comprehensive, comparative studies of the normal anterior chamber angle in major racial groups. Previous studies of the angle have used biometric measurement of the limbal or axial anterior chamber depth or an angle grading system which is primarily based on the angular width without describing the position of iris insertion and the peripheral configuration of the iris. 1. $^{4.8-12}$

This study was designed to determine and compare,

From: 'Loma Linda University Medical Center. Loma Linda,

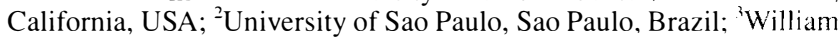
and Anna Goldberg Glaucoma Service and Research Laboratories/ Jefferson Medical College, Philadelphia, Pennsylvania, USA: ${ }^{+}$Tulane University School of Medicine, New Orleans, Louisiana, USA.

Correspondence to: George L. Spaeth, MD, William and Anna Goldberg Glaucoma Service, 900 Walnut Street, Philadelphia, PA 19107-5599, USA

Eye (1994) 8, 104-108 C 1994 Royal College of Ophthalmologists using a standardised system of gonioscopic measurement, the characteristic configuration of the anterior chamber angle in non-diseased eyes of three racial groups - AfroAmericans, Caucasians and Far East Asians - and to evaluate sexual differences and age-related changes in these same eyes. A standardised gonioscopic grading system which has been developed and used by the Glaucoma Service at Wills Eye Hospital for over 20 years was used for this study. This grading system is more comprehensive than the previously used methods. ${ }^{13.14}$ A separate study on 82 patients was designed to confirm the reliability of this grading system.

\section{METHODS}

The plan of this study was to examine approximately 100 age-matched individuals from each of three racial groups: Afro-Americans, Caucasians and Far East Asians. Subjects were recruited from healthy individuals who came to the general ophthalmology clinic at Wills Eye Hospital for routine eye examination with no previously known ocular diseases. Given an estimated rate of $0.05 \%$ angle-closure glaucoma in whites, ${ }^{7}$ the number of study subjects needed to detect a fourfold risk in other ethnic groups would be 100 in each group, given an alpha error of 0.05 (two-sided) and a beta error of 0.20 .

Two hundred and ninety-one patients were examined by two examiners (Y.G.O. and S.M.) in the following manner. Identifying data included age, sex and race. Racial classification of each patient was determined on the basis of self-description and the observer's identification. A brief history was taken, especially any previous ocular problem and family history of glaucoma or other ocular disease. Ocular examination consisted of visual acuity, intraocular pressure measurement with a Goldmann tonometer, slit lamp examination of the anterior segment, gonioscopy, and optic disc appearance. Refractive error was determined by refraction or by checking current spectacles. The ocular fundi were examined with a direct ophthalmoscope, noting the cup-to-disc ratio of the optic nerve. Gonioscopy was performed with a Zeiss four- 


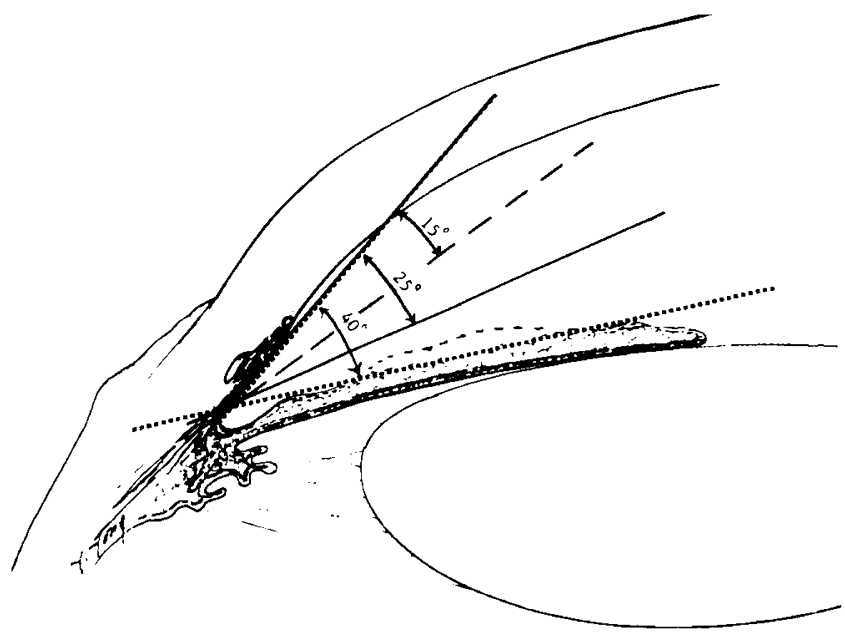

Fig. 1. The angular width of the anterior chamber recess is estimated by constructing a tangent to the anterior surface of the iris about one-third of the distance from the most peripheral portion of the iris.

mirror lens, examining $360^{\circ}$ of the angle of each eye with and without corneal indentation. The gonioscopic grading system described by Spaeth was employed in this study. ${ }^{13,14}$ The configuration of the angle was graded by three aspects: (1) the angle width, which was determined at a point slightly central to a line dropped directly posteriorly from Schwalbe's line, was measured without indentation, usually in $10^{\circ}$ increments (see Fig. 1); (2) the peripheral configuration of the iris was described before corneal indentation as 's' (steep convex curve), ' $r$ ' (regular curve without significant arching) or 'q' (concave curve) (see Fig. 2); (3) the point where the iris joins the scleral wall was graded as 'A' (anterior to the trabecular meshwork), 'B' (the area of the trabecular meshwork behind Schwalbe's line), 'C' (at the level of the scleral spur), 'D' (a deep angle recess with visible anterior ciliary body) or 'E' (extremely posterior part of the ciliary body) as shown in Fig. 3. All angles were examined and classified before and after corneal indentation using a Zeiss four-mirror lens to determine the actual position of 'iris insertion'. The degree of pigmentation in the trabecular meshwork and

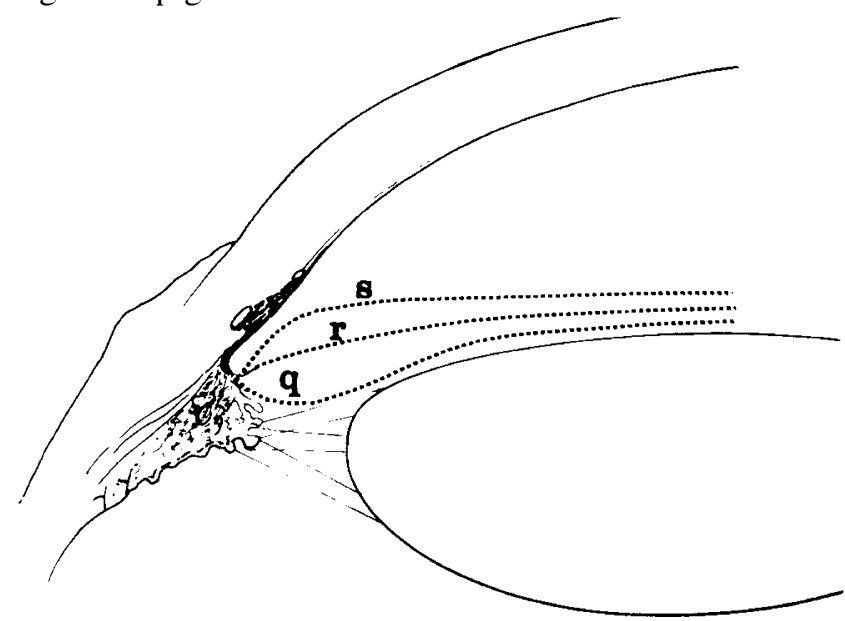

Fig. 2. The curvature of the peripheral iris is graded as: $q$, concave contour of the peripheral iris; $r$, regular curvature; $s$, steep, anterior bowing of the peripheral portion of the iris.

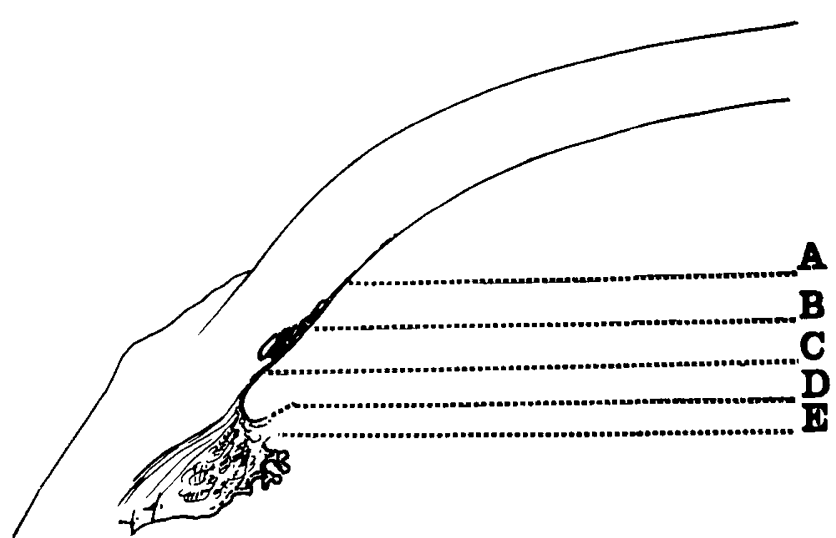

Fig. 3. Schematic drawing of locations where the iris may contact the inner side of the globe. A, iris insertion into or anterior to Schwalbe's line; B, just anterior to the posterior trabecular meshwork; $C$, scleral spur; $D$, anterior portion of the ciliary body; E, posterior portion of the ciliary body.

any peripheral anterior synechiae were described. All patients had angles examined for $360^{\circ}$ in both eyes, and the measurements at 12.00 and 6.00 o'clock in both eyes were tabulated. Because of the similarity between two eyes in each patient in this study, only the right eye was used for comparison between groups. Gonioscopy was performed by two observers (S.M. and Y.G.O.) who were each masked to the findings of the other in order to verify the accuracy of the measurement.

A study on inter-observer variability was performed on a separate group of 82 patients, using the same gonioscopic grading system. All the eyes were independently examined by two observers (G.L.S. and Y.G.O.) who were each masked to the findings of the other. These 82 patients were consecutive cases referred to a glaucoma consultation practice, and were not included in the main study because of their pre-existing ocular problems.

\section{Statistical Analysis}

Descriptive statistics for each variable were calculated for both sexes and all racial groups. Student's $t$-test and the chi-squared test were used analyse differences in mean value and proportions between groups. The correlations between refractive error and age and its dependent variables were analysed by calculating the coefficient of correlation and its $p$ value.

\section{RESULTS}

Three hundred and two patients were examined. After patients with known ocular diseases or previous history of ocular surgery had been excluded, 291 patients were included in the study. There were 97 Afro-Americans, 100 Caucasians and 94 Far East Asians (180 females and 111 males). The age, sex and refractive error of each group are listed in Table I. There were 38 blue-eyed, 36 hazel-eyed and 26 brown-eyed patients in the Caucasian group.

\section{Angular Width}

The mean angular widths at 12.00 and 6.00 o'clock are 
Table I. Comparison between ethnic groups

\begin{tabular}{llll}
\hline & Caucasian & Afro-American & Asian \\
\hline Total no. & 100 & 97 & 94 \\
Sex & F 61 & F 65 & F 54 \\
& M 39 & M 32 & M 40 \\
Age (yr) & $57.1 \pm 17.0$ & $49.9 \pm 18.0$ & $47.5 \pm 15.4$ \\
Range & $20-83$ & $13-89$ & $11-85$ \\
Female & 57.7 & 49.6 & 49.0 \\
Male & 56.3 & 49.5 & 45.4 \\
Refractive error & $+0.28 \pm 1.93$ & $-0.04 \pm 2.47$ & $-1.13 \pm 2.48$ \\
\hline
\end{tabular}

F, female; M, male

summarised in Table II. There were no statistically significant differences of the mean angular width between these groups; however, 1 Afro-American and 2 Asian patients had 'occludable' angles with peripheral anterior synechiae while there was no 'occludable' narrow angle among the Caucasians. A significant sexual difference in the angular width existed, with a mean angle width of $31.7^{\circ}$ for females and $33.8^{\circ}$ for males $(p=0.002)$. The angle also became narrower with increasing age in all ethnic groups, with a correlation coefficient of $0.488(p=0.0001)$. The refractive error had an inverse relationship with the angle width, with a correlation coefficient of 0.2478 $(p=0.0001)$.

\section{The Position of Iris Insertion}

The position of iris insertion in relation to sex and race is demonstrated in Table III. The iris joined the scleral wall most anteriorly in Asians, slightly more posteriorly in Afro-Americans and most posteriorly in Caucasians $(p=0.03)$. This racial difference was not as apparent before indentation gonioscopy, but became obvious when the actual point where the iris joins the scleral wall was determined with indentation gonioscopy. There were some sexual differences in iris insertion, with a more anterior position of the iris root in females, as shown in Table IV; however, the difference was not statistically significant $(p=0.13)$. The refractive error was positively related to the position of iris insertion $(p=0.003)$ with a more anterior insertion of the iris for hyperopic eyes (Table V). The position of iris insertion did not appear to be related to the colour of the iris.

\section{Peripheral Curve}

The irides of Asians had a greater tendency to a concave configuration of the peripheral iris compared with Caucasians and Afro-Americans ( $p=0.02$ ) (Table VI).

\section{Inter-observer Variability of Gonioscopy}

The study of inter-observer variability on a separate group

Table II. Comparison of the angular width in different ethnic groups

\begin{tabular}{llll}
\hline Angle width & Caucasian & Afro-American & Asian \\
\hline At 12.00 o'clock & $32.5 \pm 5.5$ & $31.8 \pm 5.5$ & $33.4 \pm 6.8$ \\
Range & $20-40$ & $15-40$ & $10-40$ \\
Female & $31.7 \pm 5.8$ & $30.9 \pm 5.6$ & $32.5 \pm 6.5$ \\
Male & $33.6 \pm 4.8$ & $33.6 \pm 5.0$ & $34.4 \pm 6.9$ \\
At 6.00 o'clock & 32.0 & 31.7 & 33.6 \\
\hline
\end{tabular}

There was no significant difference in angular width between ethnic groups $(p>0.24)$.
Table III. The position of iris insertion in different ethnic groups

\begin{tabular}{lccr}
\hline Classification & Caucasian & Afro-American & Asian \\
\hline Before indentation & & & \\
B & $2(2 \%)$ & $2(2 \%)$ & $3(3 \%)$ \\
C & $25(26 \%)$ & $30(31 \%)$ & $30(32 \%)$ \\
D & $70(69 \%)$ & $64(66 \%)$ & $59(63 \%)$ \\
E & $3(3 \%)$ & $1(1 \%)$ & $2(2 \%)$ \\
With indentation & & & \\
B & 0 & 0 & 0 \\
C & $10(10 \%)$ & $17(18 \%)$ & $25(27 \%)$ \\
D & $80(80 \%)$ & $68(71 \%)$ & $65(69 \%)$ \\
E & $10(10 \%)$ & $11(11 \%)$ & $4(4 \%)$ \\
\hline
\end{tabular}

B, at trabecular meshwork; C. at scleral spur: D, at anterior ciliary body; E, at mid ciliary body.

Iris insertion was most anterior in Asians, slightly more posterior in Afro-Americans. and most posterior in Caucasians $(p=0.03)$.

of 82 patients showed that the observers agreed on the location of iris insertion in $86 \%$ of the eyes examined. All differences of observation occurred in eyes in which the iris inserted close to the scleral spur, making it difficult for the observer to decide between ' $\mathrm{C}$ ' and ' $\mathrm{D}$ '. There was 99\% agreement on the angular width. The observers independently agreed on the configuration of the peripheral iris in $94 \%$ of eyes.

\section{DISCUSSION}

It is well known that angle-closure glaucoma is more common among certain racial groups. ${ }^{5-7.11}$ The incidence of angle-closure glaucoma among Caucasians over the age of 40 years is around $0.1 \%$, which comprises $6 \%$ of all glaucoma patients. ${ }^{4.6 .7}$ The incidence of angle closure glaucoma in Far East Asians is approximately 1\%, which accounts for more than $50 \%$ of all cases of glaucoma in this population. ${ }^{15-17}$ Eskimos have the highest reported incidence of 3\%.+. Angle-closure glaucoma also occurs more frequently in females than in males, ${ }^{1.11 .18}$ and the incidence increases with increasing age and with hyperopia. ${ }^{1.11 .12 .14}$

The ocular biometric traits play significant roles in the pathogenesis of angle-closure glaucoma. Eyes with angleclosure glaucoma differ significantly from normal eyes with regard to central depth of anterior chamber, axial length, corneal diameter and curvature, limbal chamber depth, relative position of the lens, and ratio of lens thickness to axial length. ${ }^{1.39 .19-21}$ In particular the anterior chamber depth is shallower in angle-closure glaucoma patients and their relatives. ${ }^{3}$ The same trend has been

Table IV. The position of iris insertion in relation to sex

\begin{tabular}{lrrrrr}
\hline & \multicolumn{2}{c}{ Before indentation } & & \multicolumn{2}{c}{ With indentation } \\
\cline { 2 - 3 } \cline { 6 - 7 } Classification & Female & Male & & Female & Male \\
\hline B & $5(3 \%)$ & $2(2 \%)$ & & 0 & 0 \\
C & $58(32 \%)$ & $25(22 \%)$ & $35(19 \%)$ & $12(11 \%)$ \\
D & $114(63 \%)$ & $81(73 \%)$ & $126(73 \%)$ & $86(77 \%)$ \\
E & $3(2 \%)$ & $3(3 \%)$ & & $14(8 \%)$ & $13(12 \%)$ \\
\hline
\end{tabular}

B, at trabecular meshwork; C. at scleral spur; D, at anterior ciliary body; E. at mid ciliary body.

There was no significant difference in iris insertion between the sexes $(p=0.13)$. 
Table V. The position of iris insertion in relation to refractive error

\begin{tabular}{lcc}
\hline Classification & Total no. & Mean refractive error \\
\hline B & 7 & $0.3 \pm 2.0$ \\
C & 85 & $0.1 \pm 2.2$ \\
D & 193 & $-0.3 \pm 2.4$ \\
E & 6 & $-1.8 \pm 2.2$ \\
\hline
\end{tabular}

$\mathrm{B}$, at trabecular meshwork; C, at scleral spur; D. at anterior ciliary body; E, at mid ciliary body.

The refractive error was positively related to the position of iris insertion, with more anterior insertion for hyperopic eyes $(p=<0.003)$.

demonstrated in females, older populations, hyperopes, and in certain ethnic groups such as Eskimos and Far East Asians. ${ }^{1.24 .11 .20-23}$ The ocular dimension deviates conspicuously towards the level that is characteristic of angleclosure glaucoma patients. ${ }^{4}$ The sexual and ethnic differences in incidence of angle-closure glaucoma correspond remarkably well with the difference in anterior chamber depth distribution. ${ }^{4}$ The shallowing of the anterior chamber with inceasing age was also confirmed by the studies of a full age range of population. ${ }^{1.2 .411 .21 .22}$ Females and hyperopes have smaller corneal diameter and limbal chamber depth and a greater ratio of lens thickness to axial length than a control population. ${ }^{8.922}$ The angle width, determined gonioscopically, correlates well with biometric traits such as lens thickness, axial length and anterior chamber depth. Our study confirms the findings of others that angle width is narrower in females than in males, and becomes narrower with increasing age. ${ }^{12.1+26}$

The underlying mechanism for the high incidence of angle-closure glaucoma, especially 'creeping' angleclosure, among Asians and blacks is not clear. The thickness and rigidity of the iris of Asians and blacks have been postulated as possible causes for their tendency towards gradual angle-closure. In our study we also found that the iris joined the ciliary body more anteriorly in Asians, even though their mean age was younger and they were more myopic than the other two groups (myopics are expected to have deeper angles). Anterior insertion of the iris can be another causative factor which may predispose to progressive formation of peripheral anterior synechiae. It has been shown that the iris tends to join the ciliary body more anteriorly in hyperopic eyes. ${ }^{13.27}$ However, there is a high prevalence of myopia among Asians, and chronic angleclosure glaucoma was reported in a group of black myopes. ${ }^{28}$ So even in the presence of a deep anterior chamber in myopic eyes, anterior insertion of the thick, rigid irides may predispose these patients to gradual angle closure.

Table VI. The peripheral curvature of the iris in different ethnic groups

\begin{tabular}{lccc}
\hline & Caucasian & Afro-American & Asian \\
\hline $\mathrm{s}$ & 2 & 1 & 0 \\
$\mathrm{r}$ & 96 & 91 & 82 \\
$\mathrm{q}$ & 2 & 5 & 12 \\
\hline
\end{tabular}

There was no statistically significant difference in the angular configuration between racial groups $(p=>0.02)$.

$\mathrm{s}$, Steep anterior bowing of the peripheral portion of the iris; r, regular curvature; q, concave contour of the peripheral iris.
In clinical practice it is not feasible to perform ultrasonography or pachymetry to determine objectively the status of the anterior chamber. Furthermore, the correlation between the biometric characteristics of the anterior chamber and those of the angle is not absolute. Most clinicians rely on gonioscopy to estimate the risk of development of angle-closure glaucoma by assessing the status of the angle. An accurate characterisation of the anterior chamber angle requires description of several different attributes of the angle.

Previous grading systems ${ }^{12.24}$ adopted by other studies classify the angular width of the anterior chamber angle but do not assess the depth of the angle, the peripheral iris configuration or the position of iris insertion, all of which are included in the Spaeth Classification used in this project. ${ }^{13.14}$ The diagnostic accuracy and consistency of this grading system are demonstrated by close agreement between the findings of the different examiners.

This study confirms several findings of previous investigations: (1) the angle becomes narrower with increasing age in all racial groups, (2) the angle in females is significantly narrower than that in males, (3) refractive error is significantly related to the width of angle. New findings include: (1) the relationship between refractive error and the position of iris insertion, and (2) the relationship between racial origin and iris insertion. There may be a direct relationship between the anatomical configuration of the anterior chamber angle, most specifically the differing anterior-posterior position of iris insertion, and the incidence of angle-closure glaucoma in these populations.

This study was supported in part by the Research Foundation of the Glaucoma Service of Wills Eye Hospital.

Key words: Angle configuration, Glaucoma, Gonioscopy, Racial differences.

\section{REFERENCES}

1. Alsbirk PH. Anterior chamber depth in Greenland Eskimos. I. A population study of variation with age and sex. Acta Ophthalmol (Copenh)1974;52:551-63.

2. Alsbirk PH. Anterior chamber depth in Greenland Eskimos. II. Geographical and ethnic variation. Acta Ophthalmol (Copenh) 1974;52:565-79.

3. Alsbirk PH. Early detection of primary angle-closure glaucoma: limbal and axial chamber depth screening in a high risk population (Greenland Eskimos). Acta Ophthalmol (Copenh) 1988;66:556-64.

4. Alsbirk PH. Primary angle-closure glaucoma: oculometry, epidemiology, and genetics in a high risk population. Acta Ophthalmol (Copenh) 1976;54:5-31.

5. Clemmensen V, Alsbirk PH. Primary angle-closure glaucoma in Greenland. Acta Ophthalmol (Copenh) 1971;49: 47-57.

6. Drance SM. Angle closure glaucoma among Canadian Eskimos. Can J Ophthalmol 1973;8:252-4.

7. Hollows FC, Graham PA. Intraocular pressure, glaucoma, and glaucoma suspects in a defined population. Br J Ophthalmol 1966;50:570.

8. Alsbirk PH. Corneal diameter in Greenland Eskimos: anthropometric and genetic studies with special reference to primary angle-closure glaucoma. Acta Ophthalmol (Copenh) 1975;53:635-46.

9. Alsbirk PH. Limbal and axial chamber depth variations: a 
population study in Eskimos. Acta Ophthalmol (Copenh) 1986;64:593-600.

10. Lee DA, Brubaker RF, Ilstrup DM. Anterior chamber dimensions in patients with narrow angles and angle-closure glaucoma. Arch Ophthalmol 1984;102:46-50.

11. Van Rens GHMB, Arkell SM, Charlton W, Doesburg W. Primary angle-closure glaucoma among Alaskan Eskimos. Doc Ophthalmol 1988;70:265-76.

12. Van Herrick W, Shaffer FN, Schwartz A. Estimation of width of angle of anterior chamber: incidence and significance of the narrow angle. Am J Ophthalmol 1969;68: $626-9$.

13. Spaeth GL. Gonioscopy: uses old and new. The inheritance of occludable angles. Ophthalmology 1978;85:222-32.

14. Spaeth GL. The normal development of the human anterior chamber angle: a new system of descriptive grading. Trans Ophthalmol Soc UK 1971;91:709-39.

15. Suzuki M, et al. The statistical observation of the glaucoma. Acta Soc Ophthalmol Jpn 1973;77:494-500.

16. Suda K, et al. Incidence of adult primary glaucoma particularly of simple and acute glaucoma in different climatic providence's in Japan. Acta Soc Ophthalmol Jpn 1964;68: 308-16.

17. Gao DW, et al. A statistical comparison study of glaucoma in the third affiliated hospital of China Medical College and Kyushu University. Acta Soc Ophthalmol Jpn 1989;93: 458-65.

18. David R, Tessler Z, Yassur Y. Epidemiology of acute angle- closure glaucoma: incidence and seasonal variations. Ophthalmologica 1985;191:4-7.

19. Panek WC, Christensen RE, Lee DA, Fazio DT, Fox LE, Scott TV. Biometric variables in patients with occludable anterior chamber angles. Am J Ophthalmol 1990;110: $185-8$.

20. Tornquist $\mathrm{R}$. Chamber depth in primary acute glaucoma. $\mathrm{Br}$ J Ophthalmol 1956:40:421-9.

21 . Tornquist R. Shallow anterior chamber in acute glaucoma. Acta Ophthalmol (Copenh) 1953;39 (Suppl):7-67.

22. Drance SM, Morgan RW, Bryett J, Fairclough M. Anterior chamber depth and gonioscopic findings among the Eskimos and Indians in the Canadian Arctic. Can J Ophthalmol 1973;8:255-9.

23. Markowitz SN, Morin D. Angle-closure glaucoma: relation between lens thickness, anterior chamber depth and age. Can J Ophthalmol 1984;7:300-2.

24. Scheie HG. Width and pigmentation of the angle of the anterior chamber. Arch Ophthalmol 1957;58:510-2.

25. Scheie HG. Width and pigmentation of the angle of anterior chamber: a system of grading by gonioscopy. Arch Ophthalmol 1957;58:510-2.

26. Calmettes ?, et al. Study of the depth of the anterior chamber: physiologic variations with particular emphasis on ametropia. Arch Ophthalmol 1958;59:513-42.

27. Lowe RF. Aetiology of the anatomical basis for primary angle-closure glaucoma. Br J Ophthalmol 1970;54:161-9.

28. Hagan JC, Lederer CM, Jr. Primary angle-closure glaucoma in a myopic kinship. Arch Ophthalmol 1985;103:353. 